\title{
Colored scalar particles production in $p p$-collisions and possible mass limits for scalar gluons from future LHC data
}

\author{
M.V. Martynov*, A.D. Smirnov ${ }^{\dagger}$ \\ Division of Theoretical Physics, Department of Physics, \\ Yaroslavl State University, Sovietskaya 14, \\ 150000 Yaroslavl, Russia.
}

\begin{abstract}
Cross sections of the colored scalar particle production in $p p$-collisions are calculated and differential and total cross sections of the corresponding parton subprocesses are obtained. The total cross section of scalar gluon production in $p p$-collisions at the LHC is estimated and the dominant decays of scalar gluons are discussed. The production cross section of scalar gluons $F$ with masses $m_{F} \lesssim 1300 \mathrm{GeV}$ is shown to be sufficient for the effective production of these particles at the LHC.
\end{abstract}

Keywords: Beyond the SM; four-color symmetry; Pati-Salam; scalar leptoquarks; scalar gluons; scalar octets.

PACS number: 12.60.-i

The search for new physics beyond the Standard Model induced by higher symmetries (supersymmetry, left-right symmetry, etc.) is one of the modern research directions in elementary particle physics. One such symmetry possibly existing in nature is the fourcolor symmetry of quarks and leptons, where leptons are considered as the fourth color [1].

Under its own minimal unification with the symmetry of the Standard Model based on the gauge group

$$
S U_{V}(4) \times S U_{L}(2) \times U_{R}(1)
$$

(the minimal quark-lepton-symmetric model - MQLS-model [2,3]), the four-color symmetry under the Higgs mechanism of quark and lepton mass splitting predicts, in particular, new scalar particles, $S U_{c}(3)$ octets and $S U_{L}(2)$ doublets, the so-called scalar gluons $F_{a^{\prime} i}$ $\left(a^{\prime}=1,2, i=1 . .8\right.$ are $S U_{L}(2)$ and $S U_{c}(3)$ indices respectively) [2-4].

By virtue of their Higgs origin, these particles have quark coupling constants proportional to the ratio of quark masses to vacuum expectation value of SM $\eta$ and, hence, the values of these constants are known (correct to within the quark mixing parameter), thus determining their interaction with gluons by the known strong coupling constant $g_{s t}$. This makes it possible to quantitatively estimate the cross sections of processes in which these particles participate. Estimates of contributions by the scalar gluon doublets $F_{a^{\prime} i}$ to the Peskin-Takeuchi S-,T-,U-parameters of radiative corrections, and their comparisons with

\footnotetext{
*E-mail: martmix@mail.ru

${ }^{\dagger}$ E-mail: asmirnov@univ.uniyar.ac.ru
} 
experimental data on $\mathrm{S}-, \mathrm{T}-, \mathrm{U}$ show that these scalar gluons may be relatively light (with masses of order $1 \mathrm{TeV}$ or less) [5,6]. Scalar gluons with such masses, being colored objects of the $S U_{c}(3)$ group, can be pair produced in $p p$-collisions through gluon fusion and, partly, through quark-antiquark pair annihilation. The scalar $S U_{c}(3)$-octets with natural suppression of FCNC have been considered in Ref. [7]. The production of scalar $S U_{c}(3)$ octets at LHC were considered in Refs. [7-10]. The light colored scalar octets in context of Adjoint $S U(5)$ unification have been discussed in Ref. [11]. In the case of the scalar gluons $F_{a^{\prime} i}$ of MQLS-model the dominant decays of these particles are known [12,13], which gives the possibility of search for these particles through their decays at LHC.

In the present paper we calculate the production cross sections of scalar particles of an arbitrary color representation in $p p$-collisions, and estimate these cross sections for

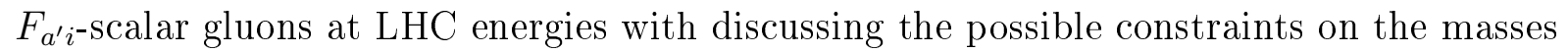
of these particles from future LHC data with account of their dominant decay modes.

Interactions of colored scalar particles $\Phi_{i}$ with gluons is contained in lagrangian

$$
\begin{gathered}
\mathcal{L}_{\Phi \Phi g}=\sum_{\text {scalars }}\left[\left(D_{i j}^{\mu} \Phi^{j}\right)^{\dagger}\left(D_{\mu}^{i k} \Phi_{k}\right)-M_{\Phi}^{2} \Phi^{i \dagger} \Phi_{i}\right], \\
D_{\mu}^{i j} \Phi_{j}=\left(\partial_{\mu} \delta^{i j}-i g_{s} G_{\mu}^{a} T_{a}^{i j}\right) \Phi_{j},
\end{gathered}
$$

where $T_{a}^{i j}$ are the generators of the group representation $S U_{c}(N)\left(a=1,2 . . d_{A}, d_{A}\right.$ - dimension of the adjoint representation of $S U_{c}(N)$ ), realized by the multiplets $\Phi_{i}, i, j$ - color index; in particular (for $N=3$ ) $i, j=1,2,3$ for scalar leptoquarks and $i, j=1,2, . .8$ for scalar gluons. The interactions of scalar leptoquark and scalar gluon doublets with fermions in MQLS-model can be found in Refs. [12,13].

In $p p$-collisions colored particles can be pair produced through gluon fusion and through quark-antiquark pair annihilation. Contributions to the total production cross section for $\Phi \Phi^{*}$ pairs are given by the parton subprocess diagrams shown in Fig. 1, 2. Diagrams of the type 2-b give a small contribution to the cross section since the corresponding constants for scalar-fermion interactions with ordinary quarks and leptons in MQLS are small $[12,13]$.

In nonabelian gauge theories the amplitudes of the scattering processes $2 \rightarrow 2$ can be represented as the product of group and Lorentz factors [14]. For the $\Phi^{*} \Phi$ production amplitude in the process of gluon fusion this gives

$$
M\left(g^{a} g^{b} \rightarrow \Phi_{i}^{*} \Phi_{j}\right) \equiv M_{i j}^{a b}=\sum_{p} g_{s t}^{2}\left(G_{p}\right)_{i j}^{a b} \frac{A_{p}}{C_{p}},
$$

where $C_{p}$ are the denominators of the corresponding propagators ( $p=s, t, u$ is the channel index, $\left.C_{s} \equiv \hat{s}=s, C_{t} \equiv \hat{t}=t-M_{\Phi}^{2}, C_{u} \equiv \hat{u}=u-M_{\Phi}^{2}\right) ;\left(G_{p}\right)_{i j}^{a b}$ are the group factors $\left(G_{t}\right)_{i j}^{a b}=\left(T^{a} T^{b}\right)_{i j},\left(G_{u}\right)_{i j}^{a b}=\left(T^{b} T^{a}\right)_{i j},\left(G_{s}\right)_{i j}^{a b}=i f_{a b c} T_{i j}^{c}\left(a, b=1 . . d_{A}\right.$ are the color indices of colliding gluons); and $A_{p}$ is the Lorentzian part of the amplitude.

The diagrams 1-a and 1-b (fig. 1) contribute to the $u$-and $t$-channels of formula (4) whereas the diagram 1-c gives the contribution to each of these channels due to the group factor of diagram 1-c $G_{t}+G_{u}$. Calculating the Lorentzian part of the amplitude gives

$$
\begin{aligned}
A_{s}= & -\left(q_{1}-q_{2}\right) \cdot \varepsilon_{1}\left(q_{1}, \sigma_{1}\right)\left(2 p_{1}+p_{2}\right) \cdot \varepsilon_{2}\left(q_{2}, \sigma_{2}\right)+ \\
& +\left(q_{1}-q_{2}\right) \cdot \varepsilon_{2}\left(q_{2}, \sigma_{2}\right)\left(2 p_{2}+p_{1}\right) \cdot \varepsilon_{1}\left(q_{1}, \sigma_{1}\right)+(\hat{u}-\hat{t}) \varepsilon_{1}\left(q_{1}, \sigma_{1}\right) \cdot \varepsilon_{2}\left(q_{2}, \sigma_{2}\right), \\
A_{t}= & \left(2 q_{1}-p_{1}\right) \cdot \varepsilon_{1}\left(q_{1}, \sigma_{1}\right)\left(p_{2}-2 q_{2}\right) \cdot \varepsilon_{2}\left(q_{2}, \sigma_{2}\right)-\hat{t} \varepsilon_{1}\left(q_{1}, \sigma_{1}\right) \cdot \varepsilon_{2}\left(q_{2}, \sigma_{2}\right), \\
A_{u}= & \left(2 q_{1}-p_{2}\right) \cdot \varepsilon_{1}\left(q_{1}, \sigma_{1}\right)\left(p_{1}-2 q_{2}\right) \cdot \varepsilon_{2}\left(q_{2}, \sigma_{2}\right)-\hat{u} \varepsilon_{1}\left(q_{1}, \sigma_{1}\right) \cdot \varepsilon_{2}\left(q_{2}, \sigma_{2}\right),
\end{aligned}
$$


where $q_{i}$ and $p_{i}$ are momenta of gluons and scalar particles respectively; $\varepsilon_{i}\left(q_{i}, \sigma_{i}\right)$ is the gluon polarization vector with momentum $q_{i}$ and polarization $\sigma_{i}, i=1,2$.

By virtue of the masslessness of gluons we have the relation

$$
C_{s}+C_{u}+C_{t}=0
$$

and taking into account the commutation relations of the $S U(N)$ group we have also the relation

$$
\left(G_{t}\right)_{i j}^{a b}-\left(G_{u}\right)_{i j}^{a b}=\left(G_{s}\right)_{i j}^{a b}
$$

From the explicit form of the amplitudes (5-7) it also follows that

$$
A_{t}-A_{u}=A_{s}
$$

With using of the relations (8-10) the squared amplitude (4) averaged over polarizations and colors of the initial gluons and summed over colors of the final particles can be written as

$$
\overline{|M|^{2}}=\frac{1}{4} \frac{1}{d_{A}^{2}} \sum_{\sigma_{1}, \sigma_{2}} \sum_{a, b} \sum_{i, j}\left|M_{i j}^{a b}\right|^{2}=g_{s t}^{4} \overline{|G|^{2}} \cdot \overline{\left|\frac{A_{t}}{C_{t}}+\frac{A_{u}}{C_{u}}\right|^{2}}
$$

where

$$
\begin{gathered}
\overline{|G|^{2}}=\frac{1}{d_{A}^{2}} \sum_{i, j, a, b}\left|\frac{C_{u}\left(G_{t}\right)_{i j}^{a b}+C_{t}\left(G_{u}\right)_{i j}^{a b}}{C_{s}}\right|^{2}=\frac{d_{\Phi}}{d_{A}^{2}} C_{2}(\Phi)\left(C_{2}(\Phi)-\frac{\hat{u} \hat{t}}{s^{2}} C_{2}(A)\right), \\
\overline{\left|\frac{A_{t}}{C_{t}}+\frac{A_{u}}{C_{u}}\right|^{2}}=\frac{1}{4} \sum_{\sigma_{1}, \sigma_{2}}\left|\frac{A_{t}}{C_{t}}+\frac{A_{u}}{C_{u}}\right|^{2}=2\left[1-2 \frac{s M_{S}^{2}}{\hat{u} \hat{t}}+2\left(\frac{s M_{S}^{2}}{\hat{u} \hat{t}}\right)^{2}\right],
\end{gathered}
$$

where $d_{\Phi}, d_{A}$ are the dimensions of the representation the field $\Phi$ and of the adjoint representation and $C_{2}(\Phi)$ and $C_{2}(A)$ are the corresponding eigenvalues of Casimir operator. Summing over gluon polarization, we employ the light-cone axial gauge in which there are no ghost contributions [15].

With account of (11-13) the differential and total $\Phi \Phi^{*}$ pair production cross sections in gluon fusion are

$$
\begin{gathered}
\frac{d \sigma_{g g \rightarrow \Phi \Phi^{*}}}{d t}=\frac{2 \pi \alpha_{s}^{2} d_{\Phi}}{d_{A}^{2} \hat{s}^{2}} C_{2}(\Phi)\left(C_{2}(\Phi)-\frac{\hat{u} \hat{t}}{\hat{s}^{2}} C_{2}(A)\right)\left[1-2 \frac{\hat{s} M_{\Phi}^{2}}{\hat{u} \hat{t}}+2\left(\frac{\hat{s} M_{\Phi}^{2}}{\hat{u} \hat{t}}\right)^{2}\right] \\
\sigma_{g g \rightarrow \Phi \Phi^{*}}=\frac{\pi \alpha_{s}^{2}}{6 \hat{s}} \frac{d_{\Phi} C_{2}(\Phi)}{d_{A}^{2}}\left[C_{2}(A) \beta\left(3-5 \beta^{2}\right)-12 C_{2} \beta\left(\beta^{2}-2\right)+\right. \\
\left.+\ln \left|\frac{\beta+1}{\beta-1}\right|\left(6 C_{2}(\Phi)\left(\beta^{4}-1\right)-3 C_{2}(A)\left(\beta^{2}-1\right)^{2}\right)\right]
\end{gathered}
$$

where $\beta=\sqrt{1-4 M_{\Phi}^{2} / s}$ is the velocity of scalar particle in the center of mass frame, and $\hat{s}$ is the squared energy in the center of momentum frame of the partons. Expressions (14) and (15) agree with the corresponding results of [7] and have the more simple form.

From formula (15) for the $S U(3)$ group $\left(C_{2}(A)=3, d_{A}=8\right)$ for $C_{2}(\Phi)=4 / 3$ and $d_{\Phi}=3$, we obtain the total production cross section of scalar leptoquarks $S$

$$
\sigma_{g g \rightarrow S S^{*}}=\frac{\pi \alpha_{s}^{2}}{96 s}\left[\beta\left(41-31 \beta^{2}\right)+\ln \left|\frac{\beta+1}{\beta-1}\right|\left(-\beta^{4}+18 \beta^{2}-17\right)\right],
$$


which coincides with that of Ref. [16]; and for $C_{2}(\Phi)=3, d_{\Phi}=8$, we obtain the total production cross section of scalar gluons $F_{a}^{\prime}$ in the form

$$
\sigma_{g g \rightarrow F_{a} F_{a}^{*}}=\frac{3 \pi \alpha_{s}^{2}}{16 s}\left[\beta\left(27-17 \beta^{2}\right)+3 \ln \left|\frac{\beta+1}{\beta-1}\right|\left(\beta^{4}+2 \beta^{2}-3\right)\right] .
$$

The production of the scalar particles $\Phi$ in quark-antiquark pair annihilation are described by the diagram shown in Fig,2 - the contribution of diagram 2-b from scalar leptoquark and scalar gluon doublets of MQLS - model are small). The corresponding cross sections are

$$
\begin{gathered}
\frac{d \sigma_{q \bar{q} \rightarrow \Phi \Phi^{*}}}{d \cos \theta}=\frac{C(\Phi) \pi \alpha_{s}^{2}}{9 s} \sin ^{2} \theta \beta^{3}, \\
\sigma_{q \bar{q} \rightarrow \Phi \Phi^{*}}=\frac{4 C(\Phi) \pi \alpha_{s}^{2}}{27 s} \beta^{3},
\end{gathered}
$$

here $C(\Phi)$ are the normalization constants of the generators for the representation $\Phi$ : $\operatorname{Tr}\left(T^{a} T^{b}\right)=C(\Phi) \delta^{a b}, C(\Phi)=3$ for scalar gluons and $C(\Phi)=1 / 2$ for scalar leptoquarks. For the scalar leptoquarks, expressions (18-19) coincide with the corresponding expressions in [16], but for scalar gluons they give cross sections 6 times larger (having the same masses) than those for scalar leptoquarks.

The total production cross section of scalar particles $\Phi \Phi^{*}$ in $p p$-collisions at LHC energy $\sigma_{t o t}=\sigma\left(p p \rightarrow \Phi \Phi^{*}\right)$ was calculated using the parton densities [17] in the leading order (LO) approximation with a fixed number of quark flavors. The total cross sections of scalar leptoquark production $\sigma_{t o t}=\sigma\left(p p \rightarrow S S^{*}\right)$ and that of scalar gluon production $\sigma_{t o t}=\sigma\left(p p \rightarrow F F^{*}\right)$ for LHC energy as functions of scalar particle masses are shown in Fig. 3. Here and below $F F^{*}$ denotes $F_{1} F_{1}^{*}$ or $F_{2} F_{2}^{*}$ pairs without their summing.

In particular, we have found that for scalar leptoquark and scalar gluon masses

$$
\begin{aligned}
& m_{S}=870_{-60}^{+50} \mathrm{GeV}, \\
& m_{F}=1300_{-130}^{+100} \mathrm{GeV}
\end{aligned}
$$

the corresponding cross sections are $\sigma\left(p p \rightarrow S S^{*}\right)=\sigma\left(p p \rightarrow F F^{*}\right)=0.01 \mathrm{pb}$ (the horizontal dashed line in Fig. 3), which gives the number of events with production of $S S^{*}$ or $F F^{*}$ pairs $N_{\text {events }}=100$ for a luminosity $L=10 \mathrm{fb}^{-1}$. The errors indicated in (20) and (21) arise from errors in the distribution functions [17]. The result (20) for scalar leptoquarks $S$ agrees with the estimate from [16].

For the more light masses the cross sections become larger and they are

$$
\begin{aligned}
\sigma\left(p p \rightarrow S S^{*}\right) & =(12-0.01) \mathrm{pb}, \\
\sigma\left(p p \rightarrow F F^{*}\right) & =(1000-0.01) \mathrm{pb}
\end{aligned}
$$

for $m_{S}=250-870 \mathrm{GeV}$ and for $m_{F_{a}}=250-1300 \mathrm{GeV}$ respectively.

Among all the possible fermionic decays of the scalar gluons $F_{1}, F_{2}=\left(\varphi_{1}+i \varphi_{2}\right) / \sqrt{2}$ of MQLS-model, the most probable are the decays

$$
F_{1} \rightarrow t \tilde{b}, \quad F_{2} \rightarrow t \tilde{t}\left(\varphi_{1}, \varphi_{2} \rightarrow t \tilde{t}\right)
$$

with the production of the third-generation quarks $[12,13]$. In the case where mass splitting inside the scalar doublets $\Delta m$ is small enough $\left(\Delta m<m_{W}\right)$, the modes (23) are 
dominant with widths of order of ten $\mathrm{GeVs}$ and with corresponding branching ratios close to unity $\operatorname{Br}\left(F_{1} \rightarrow t \tilde{b}\right) \approx \operatorname{Br}\left(F_{2} \rightarrow t \tilde{t}\right) \approx 1[12,13]$. If $\Delta m>m_{W}$, then the weak decays

$$
F \rightarrow F^{\prime} W
$$

are also possible with widths comparable to those of the decays (23).

Thus, observations of scalar gluons will be possible through the dominant events $t \tilde{t} b \tilde{b}$, $t \tilde{t} t \tilde{t}, W W t \tilde{t} b \tilde{b}$ (or $W W t \tilde{t} t \tilde{t}$ ). For comparison with (22) note that the SM background, for example, to the $t \tilde{t} b \tilde{b}$ events is of about $\sigma_{S M}(t \tilde{t} b \tilde{b}) \approx 8 \mathrm{pb}[18]$ (the darkened stripe in Fig. (3). So, the use of the appropriate cuts can give the possibility to detect the events arising from the decays of the scalar gluons with their masses of order (21) or below.

In conclusion, we resume the results of the work.

The cross sections of the scalar color particle production in $p p$-collisions are investigated. Differential and total cross sections of the corresponding parton subprocesses $\left(g g \rightarrow \Phi \Phi^{*}\right)$ and $\left(q \bar{q} \rightarrow \Phi \Phi^{*}\right)$ are obtained. The total cross sections of production of scalar leptoquarks $S$ and of scalar gluons $F$ in $p p$-collisions at the LHC are estimated and the dominant scalar gluon decay modes are discussed. The production cross section of scalar gluons with masses $m_{F} \lesssim 1300 \mathrm{GeV}$ is shown to be sufficient for the effective production $\left(N_{\text {events }} \gtrsim 100\right.$ for $L=10 \mathrm{fb}^{-1}$ ) of these particles at the LHC. 


\section{References}

[1] J. C. Pati and A. Salam, Phys. Rev. D 10, 275 (1974).

[2] A. D. Smirnov, Phys. Lett. B 346, 297 (1995), hep-ph/9503239.

[3] A. D. Smirnov, YaF. 58, 2252 (1995). (Physics of Atomic Nuclei 58, 2137 (1995).)

[4] A.V. Povarov and A. D. Smirnov, YaF. 64, 78 (2001). (Physics of Atomic Nuclei 64, No.1, 74 (2001).)

[5] A. D. Smirnov, Phys. Lett. B 513, 237 (2002).

[6] A.V. Povarov and A.D. Smirnov YaF. 66, No.12, 2259 (2003). (Physics of Atomic Nuclei 66, No.12, 2208 (2003).)

[7] A. V. Manohar, M. B. Wise, Phys. Rev. D 74, 035009 (2006), hep-ph/0606172.

[8] M. I. Gresham, M. B. Wise, Phys. Rev. D 76, 075003 (2007), arXiv.org:0706.0909.

[9] M. Gerbush et. al., arXiv.org:0710.3133.

[10] A. R. Zerwekh, C. O. Dib, R. Rosenfeld, arXiv.org:0802.4303.

[11] P. F. Perez, H. Iminniyaz, G. Rodrigo, arXiv.org:0803.4156.

[12] P. Yu. Popov, A. V. Povarov, A. D. Smirnov, Mod. Phys. Lett. A 20, 3003 (2005), hep-ph/0511149

[13] A. V. Povarov, P. Yu. Popov, A. D. Smirnov, YaF. 70, 771 (2007); (Physics of Atomic Nuclei 70, 739 (2007)).

[14] Z. Dongpei, Phys. Rev. D 222266 (1980)

[15] Cheng T.P., Li L.F. Gauge theory of elementary particle physics / Oxford, 1984.

[16] J. Blümlein, E. Boos, A. Kryukov, Z.Phys. C76, 137 (1997); hep-ph/9610408.

[17] S. Alekhin, Phys. Rev. D 67, 014002 (2003)

[18] B. P. Kersevan, E. Richter-Was, Comput. Phys. Commun. 149, 142 (2003), hep-ph/0201302 


\section{Figure captions}

Fig. 1. Diagrams of the colored scalar particle production in $g g$-fusion.

Fig. 2. Diagrams of the colored scalar particle production in $q \bar{q}$-annihilation.

Fig. 3. The total cross sections of pair production of scalar gluons (1) and of scalar leptoquarks (2) at LHC, $\sqrt{s}=14 \mathrm{TeV}$. The dashed lines (1), (2) denote errors arising from pdfs. The horizontal dashed line shows $\sigma_{t o t}=0.01 \mathrm{pb}$ which gives $N_{\text {events }}=100$ for a luminosity $L=10 \mathrm{fb}^{-1}$. The horizontal darkened stripe shows the SM background for $t \tilde{t} b \tilde{b}$ events. 


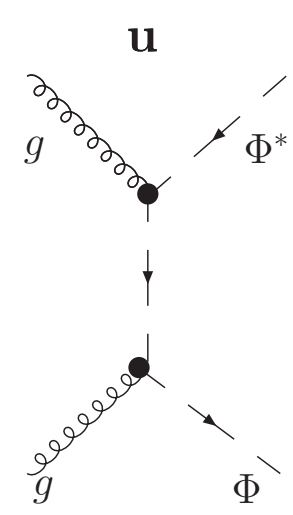

a)

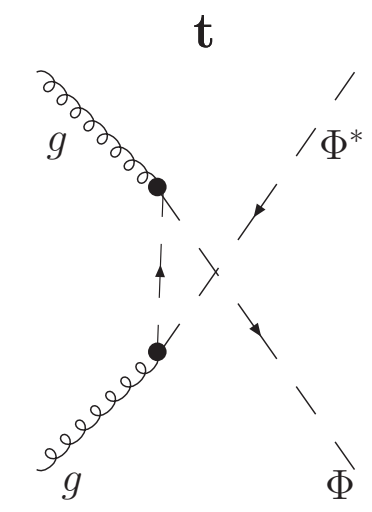

b)

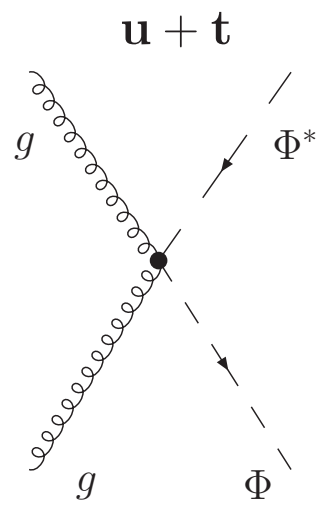

c)

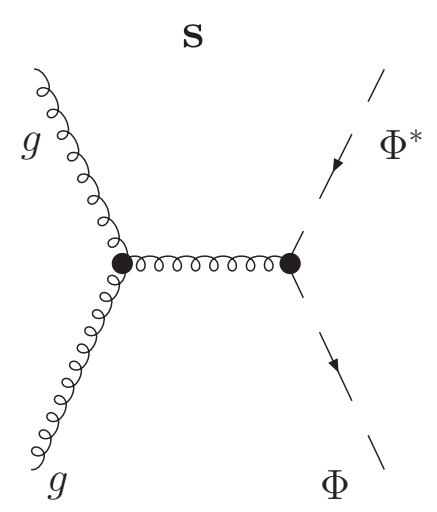

d)

Figure 1:

M.V. Martynov, A.D. Smirnov, Modern Physics Letters A

Fig. 1 

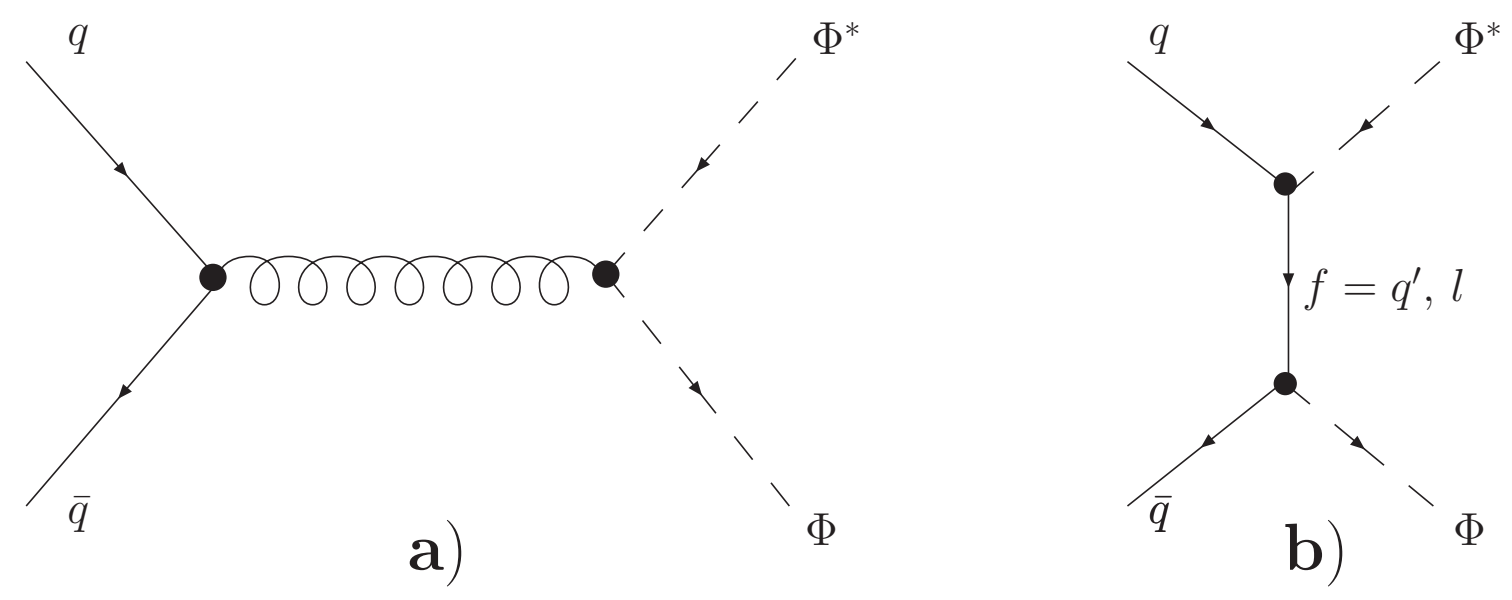

Figure 2:

M.V. Martynov, A.D. Smirnov, Modern Physics Letters A

Fig. 2 


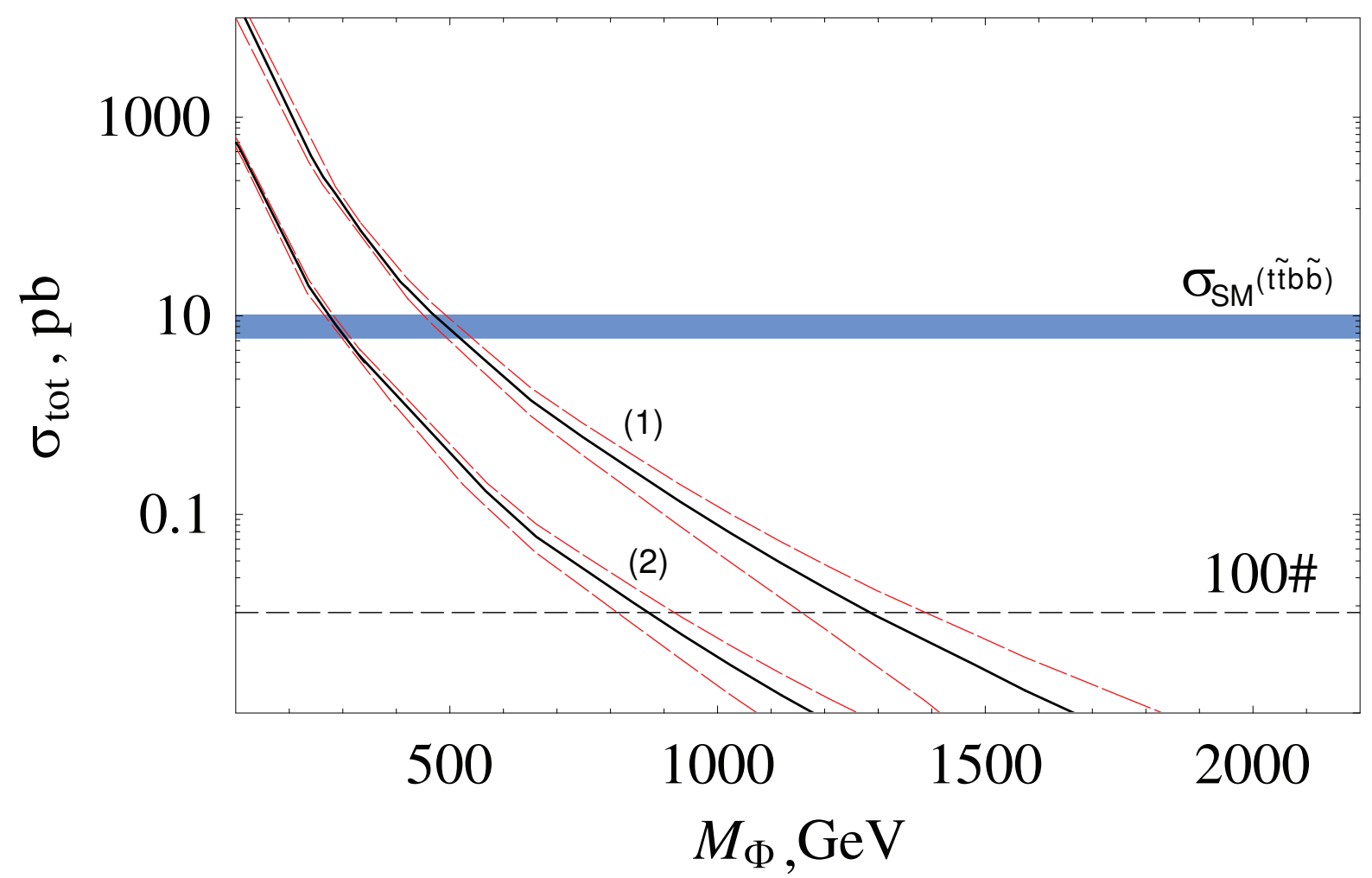

Figure 3:

M.V. Martynov, A.D. Smirnov, Modern Physics Letters A Fig. 3 